\title{
Energy-storage technologies and electricity generation
}

Professor Peter J. Hall and Dr Euan J. Bain

Department of Chemical and Process Engineering

University of Strathclyde

Glasgow

While the Office of Science and Innovation commissioned this review,

the views are those of the authors, are independent of Government and do not constitute Government policy. 
Abstract

As the contribution of electricity generated from renewable sources (wind, wave, solar) grows, the inherent intermittency of supply from such generating technologies must be addressed by a stepchange in energy storage. Furthermore, the continuously developing demands of contemporary applications require the design of versatile energy-storage/power-supply systems offering wide ranges of power density and energy density. As no single energy-storage technology has this capability, systems will comprise of combinations of technologies such as electrochemical supercapacitors, flow batteries, Lithium-ion batteries, superconducting magnetic energy storage (SMES) and kinetic energy storage. The evolution of the electrochemical supercapacitor is largely dependent on the development of optimised electrode materials (tailored to the chosen electrolyte) and electrolytes. Similarly, the development of Lithium-ion battery technology requires fundamental research in materials science aimed at delivering new electrodes and electrolytes; Lithium-ion technology has significant potential and a step-change is required in order to promote the technology from the portable electronics market into high-duty applications. Flow-battery development is largely concerned with safety and operability. However, opportunities exist to improve electrode technology yielding larger power densities. The main barriers to overcome in terms of the development of SMES technology are those related to high-temperature superconductors in terms of their granular, anisotropic nature. Materials development is essential for the successful evolution of flywheel technology. Given the appropriate research effort, the key scientific advances required in order to successfully develop energy-storage technologies generally represent realistic goals which may be achieved by 2050 .

\section{Introduction: the principles and importance of energy storage}

Energy storage and power management are becoming increasingly important as many countries are placing a greater emphasis on electrical production from renewable sources. As the contribution of electricity generated from renewal sources (wind, wave, solar) grows, the inherent intermittency of supply from such generating technologies can only be addressed by a stepchange in energy storage. Quite simply, energy-storage technologies will be vital to a future clean-energy landscape, ensuring secure and continuous supply to the consumer from a more distributed and intermittent supply base. This will be true for both on- and off-grid applications. Two billion people worldwide (third world) do not have access to electricity; they will not be supplied by a traditional national grid infrastructure. Instead, growth will be by off-grid supply, for which storage is essential, providing considerable opportunities for UK industry to supply this need, funded by the World Bank.

Table 1: Applications of energy storage

\begin{tabular}{|c|c|c|}
\hline Application & Stored capacity & $\begin{array}{l}\text { Discharge } \\
\text { period }\end{array}$ \\
\hline Grid levelling & $10 \mathrm{MJ}-200 \mathrm{GJ}$ & Seconds-days \\
\hline Power quality & $0.1-10 \mathrm{MJ}$ & Seconds \\
\hline Custom & $0.1-10 \mathrm{MJ}$ & Cycles \\
\hline Transport & $2 \mathrm{GJ}$ & Seconds-days \\
\hline
\end{tabular}

Although the need for energy storage will be far greater in the future, the problem of ensuring power quality is already upon us, as evidenced by the recent power outages in Europe and North America. It is estimated that poor power quality already causes productivity losses in the region of $\$ 400$ billion each year to the US economy. Table 1 summarises

some of the diverse applications of energy storage, illustrating the wide variation of energystorage and delivery requirements that are necessary. It illustrates the fundamental principle of energy storage - that of energy versus power. 
When the discharge period is short, devices that can deliver high power are required, for example, in overcoming fluctuations in the output of a wind turbine. Conversely, if conditions are such that there is no energy generation (calm day, solar at night), there is a requirement for a device that can store large amounts of energy and release it over what may be a long time. Unfortunately, no single device can achieve this, and to deal with the wide requirements of energy storage, an equally wide variety of technologies have been developed to cope with this. Energy storage is very much the key to unlocking the door of renewable energy.

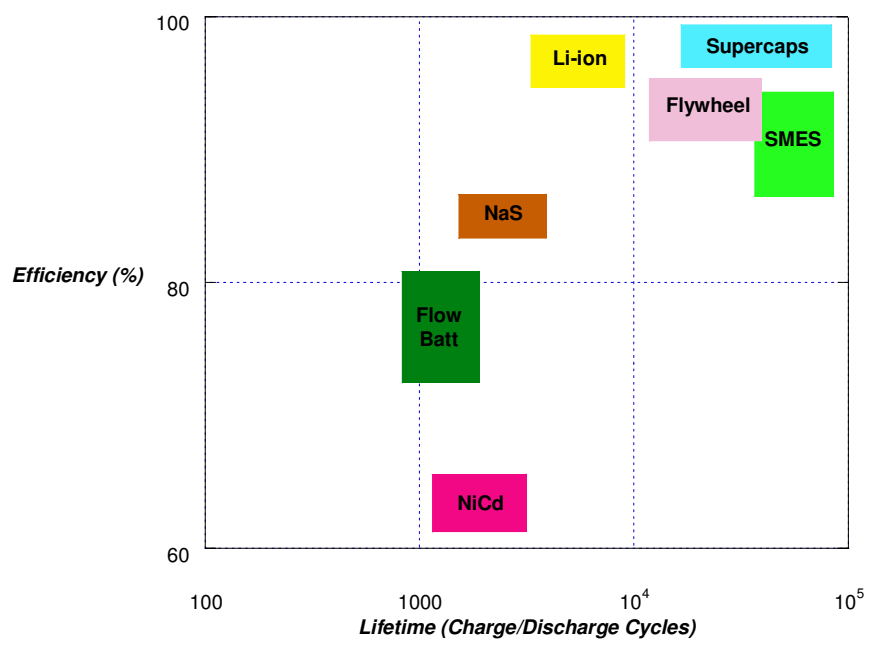

A comparison of the technologies reviewed here in terms of lifetime

Figure 1: Efficiency/lifetime properties and efficiency is presented in Figure 1. This review focuses on the scientific and engineering requirements to develop these technologies. In terms of energy storage, hydrogen is often mentioned. However, the overall efficiency of electrolyser and fuel cell is $\sim 30 \%$, making it comparable to the energy demand, for example, of a LiCd battery, but much more complicated and less safe. Hydrogen technologies are not discussed here.

\section{Electrochemical energy storage: batteries and supercapacitors}

Batteries are generally considered to represent a high-energy-density, low-power-density technology. Supercapacitors represent a high-power-density energy-storage technology which, as shown in Figure 2, is able to bridge the gap in energy density between batteries and the common capacitor.

Supercapacitors may thus be used in hybrid energy-storage systems to complement batteries and to offer periods of pulsed power which would otherwise be difficult to engineer. It is therefore essential to note that supercapacitors do not offer an alternative to batteries and that a synergy exists between the two technologies in order to meet the challenges of contemporary energy storage/power delivery systems.

\section{$2.1 \quad$ Flow batteries}

This technology essentially consists of two electrolyte reservoirs from which the electrolytes are circulated through an electrochemical cell comprising a cathode, an anode and a membrane separator. The energy density of such systems is entirely dependent on the volume of the electrolyte being stored. Power density in flow-battery systems is essentially dependent on the rates of the electrode reactions occurring at the anode and cathode respectively.

\subsubsection{Current state of technology}

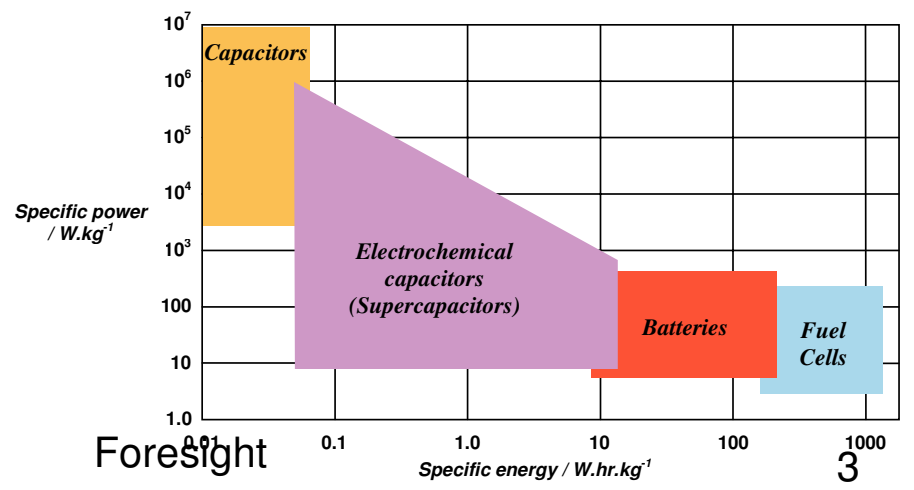

Figure 2: Ragone plot 
To date, the three leading designs of flow battery have been:

- polysulphide bromide (PSB)

- vanadium redox (VRB)

- zinc bromine $(\mathrm{ZnBr})$.

Flow batteries such as these offer energy storage/delivery efficiencies of $75-85 \%$ with potential differences, across individual cells, of $1.4-1.8 \mathrm{~V}$. Series and parallel combination of individual cells thus allow the design of high-current and high-voltage solutions. Self-discharge of flow batteries is mitigated by the isolated storage of the electrolytes in the charged state.

A major drawback of the flow battery system is the increased capital and running costs associated with the operation of a what is, in effect, a chemical plant involving pump systems, flow control with external storage. From this standpoint, the $\mathrm{ZnBr}$ system is at a disadvantage due to the necessity of a third pump system used to circulate bromine complexes.

\subsubsection{Future developments and research}

Cerium zinc $(\mathrm{CeZn})$ systems are relatively new to the market and offer larger cell potentials $(\sim 2 \mathrm{~V})$ and increased power density.

Although challenges exist in increasing energy density (via process intensification) and decreasing capital and running costs, the main challenges associated with the future development of flow-battery technology are concerned with providing increased power density via the development of monolithic electrodes with increased specific and volumetric surface areas, coupled with a high degree of wetting by electrolytes and an open pore structure to allow increased mass transfer. This may be achieved using:

- a high surface area, mesoporous carbons based on controlled porosity polymer precursors

- microreactor technology (chemical reactor on a chip) yielding high rates of mass transfer to the electrode surface, easy control of power output via scale-out, and decreased maintenance.

Developing flow batteries with increased power and energy density will increase the versatility of this technology. Flow batteries may offer an alternative to hydrogen fuel cells when coupled with wind turbines in off-grid applications.

\subsection{Lithium-ion (Li-ion), sodium sulphur ( $\mathrm{NaS})$ and related zebra battery $\left(\mathrm{Na}-\mathrm{NiCl}_{2}\right)$, nickel cadmium (NiCd) and related nickel metal hydride ( $\mathrm{Ni}-\mathrm{MeH})$, and lead acid ( $\mathrm{Pb}^{-}$acid) batteries}

Lithium-ion (Li-ion), sodium sulphur ( $\mathrm{NaS}$ ) and nickel cadmium ( $\mathrm{NiCd}$ ) batteries represent the leading technologies in high-power-density battery applications.

\subsubsection{Current status of technology}

Because the redox flow battery is a somewhat different technology, involving the flow of the reagents into the cell (more akin to a fuel cell), it has been treated in a separate section. Here, we describe the other major battery technologies, both new and old.

Of these, Liion possesses the greatest potential for future development and optimisation. Although $\mathrm{NiCd} \mathrm{Ni-MeH}$ and $\mathrm{Pb}$-acid can supply excellent pulsed power (due to their low equivalent series resistance, ESR), they are large and heavy compared to Li-ion. Nickel cadmium and lead acid also contain toxic heavy metals, which are undesirable and incompatible with a 
clean-energy future. Nickel metal hydride suffers from severe self-discharge (up to $25 \%$ per month), which means that valuable energy storage (long-term) would be lost. $\mathrm{Ni}-\mathrm{Cd}$ and $\mathrm{NiMeH}$ batteries must be fully discharged before recharge. The NaS battery, although being much smaller and lighter then $\mathrm{NiCd}, \mathrm{Ni}-\mathrm{MeH}$ and $\mathrm{Pb}$-acid, operates at $300^{\circ} \mathrm{C}$ and, although having an energy-storage efficiency of $\sim 85 \%$, requires constant heat input to maintain the molten states of the electrolytes and similar problems are encountered with the zebra battery. Li-ion batteries offer the highest energy density and need to increase the power density for some applications as well as an energy-storage efficiency close to $100 \%$. In addition, the small size and low weight of the Li-ion system makes it ideally suited to portable applications. Drawbacks Li-ion technology are its high cost and the detrimental effect that deep discharging has on its lifetime.

\subsubsection{Future developments and research}

Li-ion technology represents the highest-energy-density battery, which means it offers the greatest development potential for future application in a wide variety of energy-storage systems ranging from the fast-moving small portable electronics market to automotive and hybrid vehicles.

Lithium-ion technology has the highest energy density and therefore offers the best route for many advanced storage applications allied to clean electricity. However, the current generation of lithium devices is not sufficient for the task and a step-change is required. It is important to emphasise that this step-change will rely on fundamental research in materials science to deliver new electrodes and electrolytes.

Much of the research and development work is aimed at reducing the capital cost of Li-ion batteries through the optimisation of ancillary components such as packaging and overcharge protection circuitry. The development of new materials and nanoelectronics coupled with increases in demand suggest that it will be more viable in the future. Research is currently aimed at electrode development. The requirement of low-resistance, high-surface-area, graphitic electrodes with low resistances and good intercalation characteristics means that there are many carbon materials that offer potential for application in Li-ion systems. A $10 \mathrm{~m} \times 10 \mathrm{~m} \times 10 \mathrm{~m}$ lithium battery installation can store $400 \mathrm{MWh}$. This is comparable to a wind farm, and is a quantitative indicator of the potential significance of the technology for future clean-energy storage.

\subsection{Electrochemical supercapacitors}

Figure 2 shows that supercapacitors are essentially a high-power-density energy-storage technology. High capacitances are achieved by reducing plate separation to a few angstroms by using an electrochemical double layer and also by increasing the plate-specific surface areas to $\sim 1000 \mathrm{~m}^{2} \mathrm{~g}^{-1}$ by use of high-surface-area carbon electrodes.

\subsubsection{Current status of technology}

Supercapacitors exhibit very high energy-storage efficiencies (>95\%) and can be cycled hundreds of thousands of times without appreciable loss of energy-storage capacity. Supercapacitors therefore represent the energy-storage solution with the greatest lifetime in terms of cycling ability. Supercapacitors are, however, susceptible to self-discharge and the degree to which this affects the technology is related to the nature of the carbon electrodes. The energy $(E)$ stored by a capacitor is related to its operating potential difference $(V)$ by $E=0.5 \mathrm{CV}^{2}$. The majority of commercially available supercapacitors incorporate activated carbon electrodes and either an aqueous or organic electrolyte that may be operated at potential differences of $\sim 1 \mathrm{~V}$ and $\sim 3 \mathrm{~V}$ respectively. Organic electrolytes therefore offer increased energy density. The power density of supercapacitors is a function of the porosity, surface area and resistance of the electrode as well as the transport properties of the electrolyte. Monolithic electrodes have been shown to significantly decrease electrode resistances. Recent attention has been given to the 
development of ionic liquid electrolytes, which offer working potential differences up to $\sim 6 \mathrm{~V}$. Supercapacitors are currently applied in the portable electronics and automotive industries.

\subsubsection{Future developments and research}

The three main areas offering challenges associated with the development of carbon-based supercapacitors are as follows.

1 Electrode development:

- maximisation of wetted area and optimisation of pore diameter to suit the chosen electrolyte

- reduction of series resistance via the elimination of interparticle resistances using controlled porosity, monolithic electrodes

- development of composite electrodes, which utilise the benefits of high-surface-area carbons and electroconducting polymers.

These challenges represent achievable goals as the development of nanostructured carbons and controlled porosity polymers continues. It is likely that a large research effort will be aimed at these issues yielding a strong likelihood of success.

2 Electrolyte development:

- minimisation of fluid viscosity coupled with the maximisation of its operating potential difference.

Work on ionic liquid electrolytes is in the early stages and it is possible that such electrolytes with low viscosities at room temperature may be developed. In addition, it may be possible to use external heating to improve performance.

3 Package development:

- minimisation of weight and ESR and ensuring compatibility with other chemical species.

ESR minimisation by using monolithic electrodes has already been shown to be effective. ESR minimisation throughout the rest of the supercapacitor package is an ongoing challenge.

Although the current uses of supercapacitors are predominantly in the automotive and portable electronics industries, suitable applications also exist in the fields of medicine, defence and consumer goods. Future development will most likely see supercapacitors become integral parts of a wide variety of hybrid energy-storage/power-delivery systems.

\section{Superconducting magnetic energy storage (SMES)}

The basis of SMES is the storage of energy in the magnetic field of a DC current flowing in a superconductor. Energy losses are effectively zero because superconductors offer no resistance to electron flow. Since it is possible to inject and extract current very quickly into and out of superconducting coils, SMES has been developed for use in high-power devices. To maintain the superconducting state, the device must be cooled to the temperature at which superconductivity is attained. For low-temperature superconductors, liquid helium is necessary and, if hightemperature superconductors are used, liquid nitrogen could be used. The main advantages of SMES being used in devices for power quality are the high efficiency of storage (energy losses are associated with the cooling system but total system efficiency of $98 \%$ can be achieved), length of storage, and the devices can be cycled almost infinitely. 


\subsection{Current status of technology}

So-called micro-SMES devices (1-10 MW) are commercially available and over 30 devices with approximately $50 \mathrm{MW}$ of total capacity are installed in different parts of USA, the application being for good power quality. They are based on NbTi materials and operate at $4.2 \mathrm{~K}$. Most SMES research in the EU aims at developing micro-SMES ( $<10 \mathrm{MW})$ based on low-temperature superconducting materials. For example, the Technical University of Munich has produced a modular $1 \mathrm{MW}$ device that delivers $1 \mathrm{MW}$ operating at $3 \mathrm{kV}$ with a torus configuration. This is typical of a number of EU projects in Germany, Finland, Spain and Italy, again, based on NbTi materials operating at $4.2 \mathrm{~K}$. High-temperature superconductor technology has received much less attention but, nevertheless, successful demonstration projects operating at $20 \mathrm{~K}$ have been run in Germany, Finland, USA and South Korea.

\subsection{Future developments and research}

SMES devices may have a large variety of applications, from regulating the power output of individual renewable resources to operating in conjunction with superconducting transmission lines to provide power for large conurbations. Clearly, storage devices based on high-temperature superconducting materials will be potentially much more economic. However, there are a number of materials engineering applications to overcome. Principally, high-temperature superconductors are granular in nature. This means that grain boundaries limit the transport of current. The crystal nature of high-temperature superconductors poses problems because they are highly anisotropic. Research is also needed to increase the critical current and magnetic field for high-temperature superconductors. Improvements in this will aid the development of very-high-power-density devices. From an industrial point of view, if SMES devices based on high-temperature materials are to be widespread, research is needed on ways to produce these materials more cheaply and in much higher quantities.

The aim should be to produce SMES devices capable of $100 \mathrm{MW}$ with efficiencies of $99 \%$ and a lifetime of 40 years based on high-temperature superconductors. This could be a realistic goal by 2050. If this can be achieved, SMES will play an important role in power quality management.

\section{Kinetic energy storage: flywheels}

The basic principle of flywheel technology is that of kinetic energy stored in rotating cylinders supported by magnetic bearings and operating in a vacuum to eliminate frictional losses. They have the potential to simultaneously be both high-energy and power-density devices.

\subsection{Current status of technology}

Although flywheels with a long working lifetime (>20 years) are already available, there are no commercial applications for flywheels in power management, the technology still being at the demonstration stage. However, large arrays of flywheels have been shown to be successful in frequency management and there has been some modest success in transport applications. Research is taking place into improved bearings, using superconducting magnets and also into producing new high-tensile-strength composites capable of withstanding extremely high angular velocities. The main limitation with regard to the widespread use of flywheels is still the high cost due to the precision engineering needed.

\subsection{Future developments and research}

The major challenge is to reduce the cost of stored energy, thereby making flywheels more competitive with other forms of energy storage. There are two possibilities. The first is to produce a low-cost device with low capital and maintenance costs that could be widely deployed. Such 
devices are likely to be based on conventional materials with cheap magnetic bearings and to operate at relatively low angular velocities. The tactic here is to reduce costs by mass-producing cheap devices. The other end of the market will focus on devices that operate at much higher angular velocities. They are likely to use advanced composite materials and superconducting magnetic bearings and the tactic is to reduce costs per unit energy stored by higher performance. These devices may have different applications - cheap devices used where space is not a restriction (e.g. land-based wind turbines) and high-performance devices where physical space is at a premium (e.g. marine-based wind turbines). In addition to interfacing with renewables, flywheels could serve as energy storage for transport systems such as metropolitan railway services. 